\title{
Referenzmodell basierend auf Wertstromsimulation zur Bewertung von Produktionssystemen in der Angebotsphase
}

\author{
Markus Rabe ${ }^{1}$, Walter Wincheringer ${ }^{2}$, Tobias Sohny ${ }^{2}$ \\ ${ }^{1}$ Fachgebiet IT in Produktion und Logistik, Technische Universität Dortmund, Leonhard-Euler-Str. 5, \\ 44221 Dortmund, Germany; markus.rabe@tu-dortmund.de \\ 2Digitales Produktionslabor, Hochschule Koblenz, Konrad-Zuse-Straße 1, 56075 Koblenz, Germany; \\ wincheringer@hs-koblenz.de; sohny@hs-koblenz.de
}

\begin{abstract}
Zur Absicherung eines Angebotes von kundenindividuellen Produktionssystemen mit komplexen Materialflüssen bedarf es einer Simulation in der Angebotsphase. Basierend auf der bekannten Wertstrommethode stellt dieser Beitrag ein geeignetes Modellierungskonzept für ein Simulationsreferenzmodell vor. Es wird analysiert, welche Systemelemente für eine wertstrombasierte Simulation in der Angebotsphase unabdingbar sind. Darüber hinaus wird der Detaillierungsgrad dieser Elemente betrachtet, der (i) den Anforderungen eines begrenzten Aggregations- und Planungsaufwandes in dieser Phase und (ii) den Anforderungen, die sich aus dem dynamischen Charakter der Produktionssysteme ergeben, entspricht.
\end{abstract}

\section{Einleitung}

Anbieter von kundenindividuellen Produktionssystemen (PS) mit komplexen Materialflüssen garantieren mit der Abgabe ihres Angebots einen Mindestdurchsatz [1]. Wenn ein realisiertes PS mit komplexem Materialfluss den versprochenen Durchsatz nicht erfüllt, können hohe Kosten für den Anbieter, beispielsweise durch eine Nachbesserung, entstehen. Eine Überdimensionierung des PS schränkt die Wettbewerbsfähigkeit ein. Daher sind die Anbieter bestrebt, ihre Planung hinsichtlich des garantierten Durchsatzes bereits in der Angebotsphase abzusichern.

Zu diesem Zweck müssen die Produktionsprozesse und der Materialfluss des PS auf Auslegungsfehler analysiert werden. Die Wertstrommethode (WSM) ermöglicht hierzu eine übersichtliche Darstellung sowie deren Bewertung [2]. Allerdings können mit dieser Methode die Auswirkungen von zufällig auftretenden Ereignissen, wie z. B. stochastische Betriebsmittelausfälle, nicht abgebildet werden [3]. Die Bewertung dynamischer Interdependenzen innerhalb eines PS mit komplexem Materialfluss erfordert daher den Einsatz der zeitdiskreten Simulation [1]. Diese ermöglicht es, dynamische Systeme über die Zeit in ausführbaren Modellen zu betrachten [4].

Die Simulation ist jedoch zeitaufwendig, kostspielig und daher für die Angebotsphase nur bedingt geeignet. Eine detaillierte Planung mit Simulation beginnt daher oft erst, wenn der Auftrag bereits erteilt ist [1].

\section{Stand der Technik}

Wertstrommethode. Die Wertstrommethode (WSM) ermöglicht eine transparente Darstellung aller produktspezifischen Produktionsprozesse mit den zugehörigen Material- und Informationsflüssen in der diskreten Fertigung [2]. Für die Bewertung dynamischer Aspekte hat sich die ereignisdiskrete Simulation (discrete event simulation (DES)) als geeigneter Ansatz erwiesen [1].

Simulation. In Produktion und Logistik wird die Simulation als das „Nachbilden eines Systems mit seinen dynamischen Prozessen in einem experimentierbaren Modell [...]." [5] definiert. Ihr primäres Defizit ist die zeitaufwändige Modellierung, welche mittels Referenzmodellen (RM) auf ein vertretbares Niveau reduziert werden kann [1].

Referenzmodell. Im Bereich der Simulation dienen Referenzmodelle als Konstruktionsschemata für den Entwurf von aufgabenbezogenen Simulationsmodellen 
[6]. Ein Referenzmodell befindet sich dabei auf der gleichen semantischen Stufe wie das Modell, das mit ihm modelliert wird. Es fokussiert sich auf die Semantik und verallgemeinert die Syntax [7]. Darüber hinaus berücksichtigt ein Referenzmodell nicht die Systemarchitektur eines Simulationssystems [7]. Ein Modulelement-Baukasten eines Simulationssystems ist folglich kein Referenzmodell, sondern die simulatorspezifische Implementierung eines Referenzmodells [6].

Dynamische Wertstrommethode. Die Kombination der statischen WSM mit der DES ist daher naheliegend, jedoch keine neue Idee. Den existierenden Ansätzen ist gemein, dass die Wertstrommodelle in Simulationssysteme übertragen oder um diese ergänzt wurden. Das Ziel war jedoch, eine dynamische Lean-Methode zu erhalten [8]. Für die Sicherung der Planungsqualität in der Angebotsphase mit Wertstromsimulation ist jedoch eine höhere Granularität der Eingangsdaten erforderlich [9].

\section{Struktur des Referenzmodells}

Für die Kombination der WSM mit der DES ist es erforderlich, eine geeignete Granularität der Datenbasis und der Prozessbeschreibungen zu bestimmen. Aufbauend auf der Modellierung der WSM müssen hierzu deren Grundelemente Produktionsprozess, Material- und Informationsfluss analysiert und erweitert werden.

Produktionsprozess. Der Produktionsprozess wird in der WSM durch einen Datenkasten mit produktionsspezifischen Kennzahlen abgebildet [2]. Bestimmte vorhandene Parameter werden um die dynamischen Betrachtungen für eine Simulation ergänzt [9].

Sowohl die Zykluszeit (ZZ), als kapazitives Angebot eines Produktionsprozesses, als auch die Prozesszeit (PZ), zur Abbildung von Chargenprozessen [2], müssen produkt- und prozessspezifisch betrachtet werden.

Zur Darstellung von Haupt- und Nebenmaterialflüssen ist die Prozessmenge (PM), zur Abbildung der in einer Charge integrierten Einheiten [2], ebenfalls produkt- und prozessspezifisch zu berücksichtigen.

Auftragsbezogene Ereignisse wie Losgrößen, die eine Rüstaktion bedingen, sowie stochastische Perioden der Rüstzeit müssen bedacht werden [4].

Ein Prozentsatz zur Abbildung der Verfügbarkeit ist für eine Simulation nicht ausreichend. Zu diesem Zweck muss der Parameter um die Zeitpunkte des Ausfalls (Mean Time Between Failure - MTBF) in Form von Verteilungen wie der Exponentialverteilung [4], der Anzahl der Aktionen bis zum Auftreten eines Ausfalls oder der Ereignisse, die eine Störung verursachen, erweitert werden. Darüber hinaus muss die Reparaturdauer (Mean Time To Repair - MTTR) in Verbindung mit einer Verteilung, wie der Erlang-Verteilung, angepasst werden [4].

Zuletzt muss die produkt- und rohstoffabhängige Produktionsausbeute eines Produktionsprozesses sowie Ereignisse, welche Schlechtteile bedingen, mit einbezogen werden [4].

Abbildung 1 zeigt den statischen Prozessdatenkasten (PDK) der WSM, erweitert um die notwendigen Parameter für eine Simulation.

\begin{tabular}{|c|c|c|}
\hline Statisch & \multicolumn{2}{|c|}{ Dymamisch } \\
\hline Prozess Nr. / Name & & -Zeitpunkt des Rüstens: \\
\hline$\underbrace{\square}_{\# \text { Mitarbeiter \# Res }}$ & & $\begin{array}{l}\text { Event } \\
\text { Losgröße } \\
\text { Ruistzeit. }\end{array}$ \\
\hline Prozesszeit (PZ) & - Varianz: & Rūstzeit (Varianz) \\
\hline Prozessmenge (PM) & produktspezifisch & \\
\hline Zykluszeit (ZZ) & prozessspezifisch & -Ausfallzeitnunkt: \\
\hline Rüstzeit (RZ) & & MTBF (Varianz) \\
\hline Losgröße (LG) & -Zeitpunkt Ausschuss: & \#Aktionen \\
\hline Verfugbarkeit (V) & Event & Event \\
\hline Gutausbeute & $\frac{\text {-Menge des Ausschusses: }}{\text { produktspezifisch }}$ & $\frac{\text {-Instandsetzung: }}{\text { MTTR (Varianz) }}$ \\
\hline
\end{tabular}

Abbildung 1: PDK erweitert für die DES [9].

Materialfluss. „Der Materialfluss ist die Verkettung aller Vorgänge beim Gewinnen, Be- und Verarbeiten sowie bei der Verteilung von Gütern innerhalb festgelegter Bereiche“ [10]. Mittels der WSM wird dieser lediglich durch die Fließrichtung (Pfeilsymbol) und Materialbestände in Form von Reichweiten (Dreiecke) zwischen Produktionsprozessen dargestellt [2]. Zur Abbildung der logistischen Funktionen zur geographischen oder zeitlichen Transformation von Gütern wird ein erweiterter Datenkasten mit Kennzahlen (Key Performance Indicator - KPI) für den Transport benötigt [11].

Unter Transport ist die Ortsveränderung von Gütern mit technischen Mitteln zu verstehen. Ist diese Ortsveränderung räumlich begrenzt, beispielsweise innerhalb einer Fabrik (Intralogistik), wird dies mit dem Begriff Fördern spezifiziert und die technischen Transportmittel als Fördermittel deklariert [12].

In Bezug auf den diskreten Materialfluss von Gütern erzeugen Fördermittel einen kontinuierlichen oder diskontinuierlichen Fluss des geförderten Materials. Daher 
wird zwischen stetiger und unstetiger Förderung differenziert [12].

Analog zu den Produktionsprozessen müssen für den Materialtransport im wertstrombasierten Referenzmodell dynamische Kenngrößen erarbeitet werden, welche die Förderprozesse unabhängig davon, ob es sich um einen Stetig- oder Unstetigförderer handelt, in geeigneter Granularität abbilden. Hierzu ist eine Analyse der Stetigund Unstetigförderer erforderlich.

Stetigförderer. Stetigförderer (z.B. ein Förderband) sind mit festen Führungsschienen ausgestattet. Die Fördergüter überholen sich dabei nicht und werden nach dem FIFO-Prinzip gefördert [12]. Die Beladung und die Entladung können während des Transportprozesses erfolgen. Abhängig vom Typ dieser Förderer ist eine Stauung des Förderguts möglich [12].

Hierzu sind, in Abhängigkeit der Modellierungsaufgabe, spezifische KPIs notwendig. Abgängig von den Relationen der Zykluszeiten der Produktions- und Förderprozesse können drei Fälle definiert werden:

Für den Fall, dass die ZZ der Produktionsprozesse einen wesentlich größeren Zeitanteil als die Förderzeit aufweist (ZZ $Z_{\text {Produktionsprozess }}>>$ ZZ $Z_{\text {Förderer }}$ ), muss der Förderprozess nicht abgebildet werden, da er keinen Engpass im Materialfluss bildet. Beispielsweise ist hier ein Glühofen $(\mathrm{PZ}=2 \mathrm{~h})$ zu nennen. Die Förderzeit zum Folgeprozess ist hier mit einer PZ von vier Minuten vernachlässigbar klein und bedarf keiner weiteren Betrachtung. Grundsätzlich ist die Pufferkapazität zu betrachten, welche im Weiteren analysiert wird.

Für den Fall, dass die Zykluszeit eines Förderers wesentlich größer als die Zykluszeit des Produktionsprozesses ist ( $Z Z_{\text {Produktionsprozess }}<<Z_{\text {Förderer }}$ ), liegt ein Auslegungsfehler des Systems vor. Dieser Engpass kann durch eine zusätzliche Fördereinheit oder durch Erhöhung der Fördergeschwindigkeit beseitigt werden. Folglich wird dieser Fall nicht weiter untersucht. Auch hier ist die Pufferkapazität zu berücksichtigen.

Für den Fall, dass die Zykluszeit des Förderers nicht allzu sehr von der Zykluszeit des Produktionsprozesses abweicht, bedarf es einer detaillierten Betrachtung. Neben der Überbrückung von räumlichen Distanzen haben Förderer die Aufgabe zur Entkopplung von Produktionsprozessen [12]. Aus Materialflusssicht ist hierbei die Pufferkapazität entscheidend, welche u. a. einen temporären Taktausgleich der vor- und nachgelagerten Produktionsprozesse erlaubt. Der Puffer ist hierbei ein Anlagen- bereich (Entkopplungsmodul), welcher eine vorübergehende Pufferung von Fördergütern, um zwei Anlagenbereiche zu entkoppeln, ermöglicht [12].

Aus Wertstromgesichtspunkten handelt es sich hierbei stets um eine FIFO-Verkopplung [2]. Taktzeitdifferenzen oder Rüstzeitpuffer der zu verknüpfenden Produktionsprozesse können hiermit ausgeglichen werden [2]. Folglich kann die Anzahl an Pufferplätzen mittels der vorliegenden Taktzeitdifferenz in Form einer Zykluszeit statisch abgebildet werden (Abbildung 2).

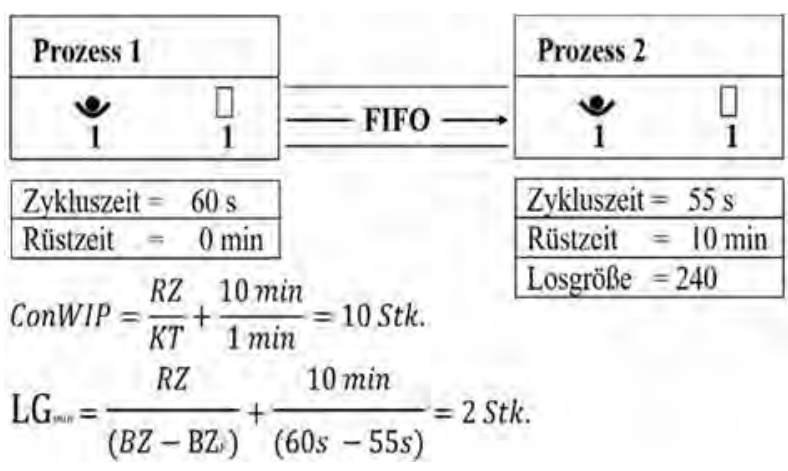

Abbildung 2: Beispiel einer FIFO-Verkopplung (nach [2])

Für einen optimalen Fluss ist somit u. a. die optimale Auslegung des Puffers eines Förderers notwendig. Stochastische Ausfälle der vor- und nachgelagerten Prozesse eines Förderers sowie eine produktspezifische ZZ erschweren diese Auslegung. Hierzu bedarf es einer Analyse der Einflussgrößen. Für den Fall, dass große Distanzen durch Förderer überbrückt werden sollen, existieren im Kontext der Pufferbetrachtung drei Szenarien:

Ist der Förderer leer (der Puffer also nicht genutzt), so fährt der Förderer mit einer Geschwindigkeit von $v_{\max }$ und die Fördergüter werden ohne Verzögerung oder Beschleunigung dem nachgelagerten System zugeführt. Entscheidend ist, wann die Fördergüter dieses nachgelagerte System erreichen. Hier bestimmt sich der Zeitbedarf zur Distanzüberbrückung durch die Förderzeit in Abhängigkeit der konstanten Fördererlänge ( $s_{\text {konst }}$ ) und der maximalen Geschwindigkeit $\left(v_{\max }\right)$ :

$$
t_{\min }=\frac{s_{\text {konst }}}{v_{\max }}
$$

Sobald der Förderer teilbefüllt ist, ergibt sich aus der Materialflusssicht die Frage, wann der nächste Artikel für den Folgeprozess zur Verfügung steht. Sobald der Folgeprozess ausgelastet ist $(\mathrm{PM}=1)$ wird maximal ein Teil 
weitergeleitet. Auf dem Fördersystem ergibt sich somit eine Stausituation. Das Fördergut erfährt hierbei eine Verzögerung $\left(a_{v}<0\right)$ bei einem Stopp sowie eine Beschleunigung $\left(a_{b}>0\right)$ bei einer erneuten Anfahrt. Daraus ergibt sich eine vereinfachte lineare GeschwindigkeitsDarstellung, die auch als Trapezfahrt bezeichnet wird (Abbildung 3) [13].

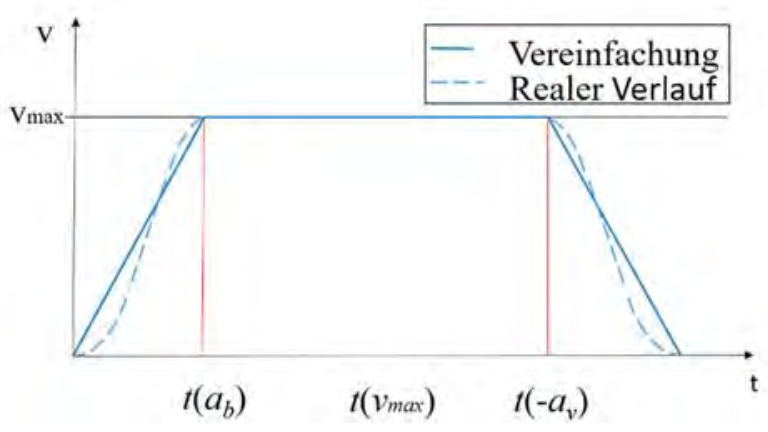

Abbildung 3: Geschwindigkeits-Zeit-Diagramm (nach [13]).

Die Förderzeit untergliedert sich dabei in drei Teilspiele: die Beschleunigungsphase $t\left(a_{b}\right)$, die konstante Fahrzeit $t\left(v_{\max }\right)$ und die Verzögerungsphase $t\left(-a_{v}\right)$.

Das reale Geschwindigkeitsprofil $v(\mathrm{t})$ weicht in der Beschleunigungs- und Bremsphase von dem vereinfachten trapezförmigen Geschwindigkeitsprofil ab (S-Kurve). Das reale Systemverhalten kann mit dem trapezförmigen Geschwindigkeitsprofil allerdings ausreichend genau angenähert werden [14]. Die gesamte Spielzeit ergibt sich somit aus der Summe der Teilspiele [13].

$$
\begin{aligned}
& t_{\text {ges }}=\mathrm{t}\left(a_{b}\right)+\mathrm{t}\left(v_{\text {max }}\right)+\mathrm{t}\left(-a_{v}\right) \\
& =\frac{s_{\text {konst }}}{v_{\text {max }}}+\frac{v_{\text {max }}}{a}
\end{aligned}
$$

Folglich fallen die Beschleunigung und Verzögerung additiv bei einem Stoppvorgang mit anschließender Anfahrt des Förderers an. Für die Ermittlung des Zeitanteils bei einer Beschleunigung und Verzögerung ist die $\mathrm{zu}$ überbrückende Distanz von Bedeutung. Ist die Wegstrecke zu kurz, kann die Nenngeschwindigkeit $v_{\max }$ (Trapezfahrt) nicht erreicht wurden. Die Beschleunigungsphase geht direkt in die Verzögerungsphase über und weist eine dreieckige $v$ - $t$-Darstellung auf (Dreiecksfahrt, Abbildung 4) [15].

Der Zeitbedarf einer Beschleunigung oder Verzögerung ist somit unabhängig von der Fördererlänge zu berücksichtigen. Entscheidend sind die durch den Folgeprozess verursachten Anzahlen an Stoppvorgängen $n$.

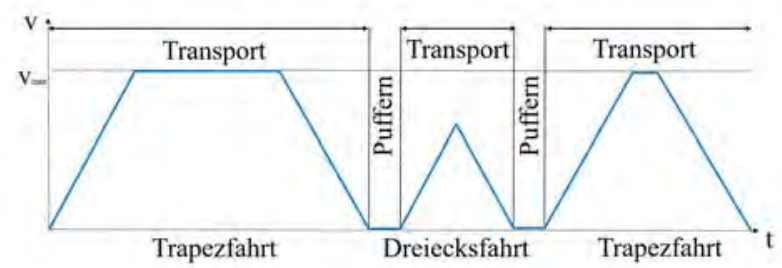

Abbildung 4: v-t-Darstellung eines Stetigförderers

$$
\begin{aligned}
& t_{\text {ges }}=\frac{s_{\text {konst }}}{v_{\max }}+n * \frac{v_{\max }}{a} \text { für } s \geq \frac{v_{\max }{ }^{2}}{a} \text { Trapezfahrt } \\
& \text { bzw. } 2 * t(a)=2 * \sqrt{\frac{s}{a}} \text { für } s<\frac{v_{\max }^{2}}{a} \text { Dreiecksfahrt } \\
& \text { mit }\{n \in \mathbb{N}\} \text { (3) [15] }
\end{aligned}
$$

Es sei angemerkt, dass Trägheitsmomente sowie ein entstehender Schlupf bei einer Anfahrt nicht berücksichtigt werden. Darüber hinaus wird $a_{b}=-a_{v}$ angenommen. Eine durch einen Stopper verursachte Verzögerung mit $-a_{v}>a_{b}$ wird nicht betrachtet.

Die zu überbrückende Distanz bei einem erneuten Anlauf ergibt sich aus der Länge des jeweiligen Förderelements plus seinem Abstand zu den folgenden Fördergütern (Abbildung 5).

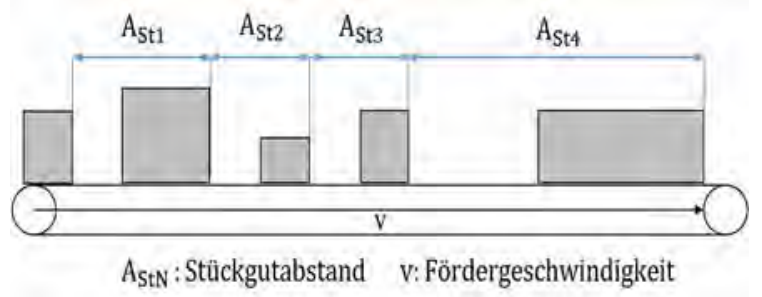

Abbildung 5: Stückgutabstand [12]

Diese ergeben den Stückgutabstand und ermöglichen die Differenzierung zwischen einer Dreiecks- und einer Trapezfahrt. Die jeweilige Fördergutlänge einschließlich des Abstandes ist produktspezifisch und muss dem jeweiligen Produkt zugeordnet werden.

Die vorangegangenen Analysen bedingen, dass der Einfluss des Stückgutabstands sowie die Geschwindigkeit und der in Abhängigkeit einer Stausituation relevante Faktor $2 t(a)$ bei einer Zuführung berücksichtigt werden müssen. Dies gilt insbesondere, wenn der vorgelagerte Prozess des Förderers eine schnellere PZ aufweist als die Fördergüter weggefördert werden. Das Fördergut kann dem System erst zugeführt werden, sobald die erste Position (abhängig vom Fördergutabstand) auf dem Förderer frei ist (Abbildung 6).

Dieser entstehende zeitliche Verlust einer Teilzykluszeit führt u. U. zu einem Materialflussabriss und ist dementsprechend zu berücksichtigen. Darüber hinaus ist die 
zusätzliche Zeitspanne, bis das Produkt das Fördersystem verlässt, zu beachten. Diese muss, in Abhängigkeit der Stückgutlänge, zur Förderzeit addiert werden. Bei einer diskreten Fertigung in der Praxis ist diese zusätzliche Zeit in der Regel vernachlässigbar klein.

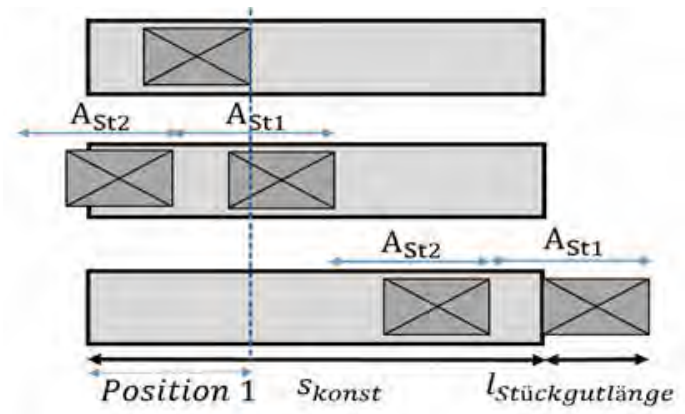

Abbildung 6: Zuführung und Abfuhr eines Förderelements

$$
t_{\text {min }+ \text { Ausschleusung }}=\frac{s_{\text {konst }}+l_{\text {stückgutlänge }}}{v_{\max }}
$$

Aus Szenario 2 ergeben sich folgende Kenngrößen:

- Fördererlänge

- Geschwindigkeit

- Beschleunigung/Verzögerung (Anzahl n)

- Artikellänge

- Stückgutabstand

- Zufuhr/Abfuhr

Sobald die Fördergüter - z. B. bedingt durch einen Ausfall des Folgeprozesses - sich bis zu dem zuführenden Prozess aufstauen, liegt eine Blockade vor. Der so entstehende Stau beschreibt die zeitliche Verzögerung eines Materialflussobjektes, bedingt durch das Warten auf die Abfertigung eines oder mehrerer sich vor ihm befindlichen Objekte [16]. Entscheidend hierbei ist die Kapazität des Förderers. Diese entspricht dem maximalen physischen Aufnahmevermögen des Förderers [13].

Die Kapazität ergibt sich aus dem Verhältnis des produktspezifischen Stückgutabstandes zu der Fördererlänge. Bedingt durch den schwankenden Stückgutabstand ergibt sich eine variable Kapazität. Diese soll folglich durch das System ausgewertet werden. Bei nicht stauenden Förderern ist diese geringer, da der Förderer stoppt sobald der Folgeprozess ausgelastet ist, was den vorgelagerten Prozess blockiert. Erst wenn der Folgeprozess frei wird, ist eine Wiederaufnahme der Förderung möglich.
Der Fördergutstau kann mittels eines Gruppen- oder sequentiellen Abzuges aufgelöst werden [12]:

Bei einem Gruppenabzug werden die Fördergüter in einem Block weitergefördert [12]. Bei einem sequentiellen Abzug werden die aufgestauten Güter unter Einhaltung des Stückgutabstandes nacheinander weitergefördert (Abbildung 7) [12].

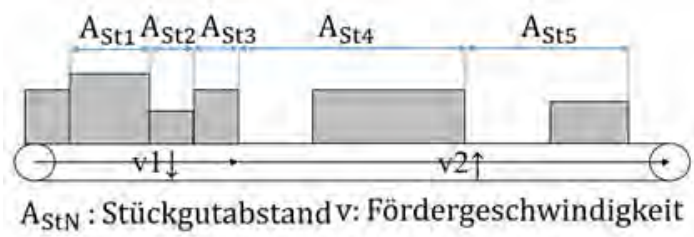

Abbildung 7: Auflösung des Materialstaus [12]

Aus Szenario 3 ergeben sich folgende Kenngrößen:

- Kapazität

- Stauauflösung (sequenzieller oder Gruppenabzug)

Unabhängig von der betrachteten Kenngröße ist die Verfügbarkeit der Förderer, wie bei den Produktionsprozessen, mittels Verteilungen abzubilden.

Ein weiterer Aspekt, welcher bei der Analyse von Stetigförderern zu berücksichtigen ist, betrifft die Verknüpfung von Förderern. Bei einem Übergang von unterschiedlichen Stetig-Fördersystemen können verschiedene Geschwindigkeiten vorliegen. Hierzu ergeben sich drei Fälle (Abbildung 8):

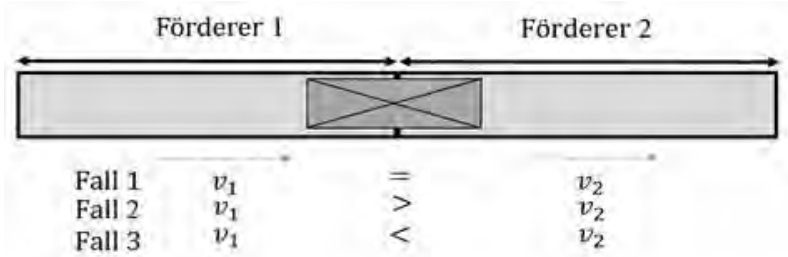

Abbildung 8: Übergang von Förderern

Der Massenschwerpunkt, Reibungskräfte sowie ein möglicher Schlupf werden nicht betrachtet. Sobald das Fördergut sich auf dem folgenden Förderer befindet, sind dessen Geschwindigkeit und Beschleunigung maßgebend.

Wenn die Geschwindigkeiten identisch sind $\left(v_{1}=\right.$ $v_{2}$ ), können die Förderer als eine Fördereinheit kombiniert werden.

Ist $v_{1}>v_{2}$, so ist $v_{2}$ beim Übergang bestimmend und verursacht einen Materialstau. Vorhandene Stückgutabstände werden bei dem Übergang reduziert. Der 
zusätzliche Zeitbedarf des spezifischen Stückgutabstands wird beim Verlassen von Förderer 2 berücksichtigt.

In dem Fall, dass $v_{1}<v_{2}$ ist, wird $v_{2}$ bestimmend für die Zuführung und das Fördergut wird beim Übergang schneller gefördert. Vorhandene Stückgutabstände werden am Übergang vergrößert. Der zusätzliche Zeitbedarf des spezifischen Stückgutabstands wird beim Verlassen von Förderer 2 berücksichtigt.

Abbildung 9 fasst die Erkenntnisse der Analyse in einem Datenkasten für Stetigförderer zusammen. Die definierten Eingabeparameter sowie die produktspezifischen Attribute werden bei der Simulation berücksichtigt, sodass die sich daraus ergebende Förderzeit und Kapazität bestimmt werden können. Zudem werden die produktspezifische Ablauflogik bei einer Anfahrt oder Stoppaktion sowie die Systemzufuhr und -abfuhr automatisch verarbeitet. Darüber hinaus bedarf es der Auswahl, ob es sich um einen stauenden oder nicht stauenden Förderer handelt sowie der Definition der Stauauflösung. Mittels des Datenkastens soll neben der Abbildung der Stetigförderer mit einer Simulation die optimale Pufferkapazität eines Förderers durch den Anwender ermittelt werden (Was-wäre-wenn-Szenarien).

\begin{tabular}{|c|c|}
\hline Förderer & Dynamisch \\
\hline Stetigförderer & Aut. Darstellung \\
\hline$\square$ & $\sqrt{\text { Fürderzeit }}$ \\
\hline \# Ressourcen & Kapavitint \\
\hline [Jalkein) & axitait \\
\hline Stauauntäsung (sequenriell grup.) & \\
\hline Fördererlänge (s) & \\
\hline Geschwindigkeit (v) & Logik \\
\hline Beschleunigung/Verzögerung (a) & \\
\hline Artikellänge & Zuvor/Ahfuhr \\
\hline Stückgutabstand & \#Anfahr-/Abbremsverhalten \\
\hline Verfügbarkeit (MTBF, MTTR) & \\
\hline
\end{tabular}

Abbildung 9: Datenkasten für Stetigförderer

Unstetigförderer. Im Vergleich zu den Stetigförderern können sich die Unstetigförderer (z. B. Fahrerlose Transportsysteme) frei bewegen und haben daher einen höheren Grad an Flexibilität. Der Be- und Entladevorgang findet statt, sobald der Unstetigförderer stoppt [12]. Der Be- und Entladevorgang kann hierbei mit dem zuvor definierten Datenkasten für Produktionsprozesse dargestellt werden [9]. Die Darstellung von Unstetigförderern wird in weiteren Forschungsansätzen analysiert. Neben seiner Kapazität und Verfügbarkeit spielt die Förderzeit eine entscheidende Rolle. Basierend auf der Geschwindigkeit und Beschleunigung ist diese abhängig von der Quellen- und Senkenposition und dem sich daraus ergebenden Pfad. Zur Darstellung ist die Definition einer in der Praxis üblichen Transportmatrix, welche die Abbildung aller Fahrdistanzen ermöglicht, denkbar [17]. Potentielle Verzögerungen wie das Stoppverhalten durch Mitarbeiter auf der Fahrbahn oder Vorfahrtsregeln an Kreuzungen lassen sich in einer frühen Angebotsphase durch Erfahrungsgrößen berücksichtigen [18]. Aus der Materialflusssicht ist allerdings die Anzahl an FTS relevant, sodass der Fluss nicht abreißt. Ein einzelnes Fahrzeug ist bei einer solchen frühen Planung nicht ausschlaggebend. Entsprechende Kenngrößen für die ausreichend genaue Abbildung von Unstetigförderern sind zu erarbeiten und in Form eines Datenkastens darzustellen.

I nformationsfluss. Für eine Kombination der Produktions- und Transportprozesse bedarf es einer logistischen Verknüpfung. Innerhalb der WSM werden hierzu die Informationsflüsse visuell abgebildet. Für eine DES betrachten die WSM und die bestehenden Ansätze der Wertstromsimulation die Materialflusslogik zu oberflächlich [19]. Parallel ablaufende Prozesse, stochastische Einflüsse und Montageprozesse mit verschiedenen Produkten werden nicht beachtet. Hierzu bedarf es einer Berücksichtigung der Materialflusslogik, sodass Produktionsprozesse untereinander oder mit diversen Förderern verknüpft werden können. Hierzu werden die Grundelemente der WSM um die Logik, welche genau dies ermöglichen soll, erweitert (Abbildung 10).

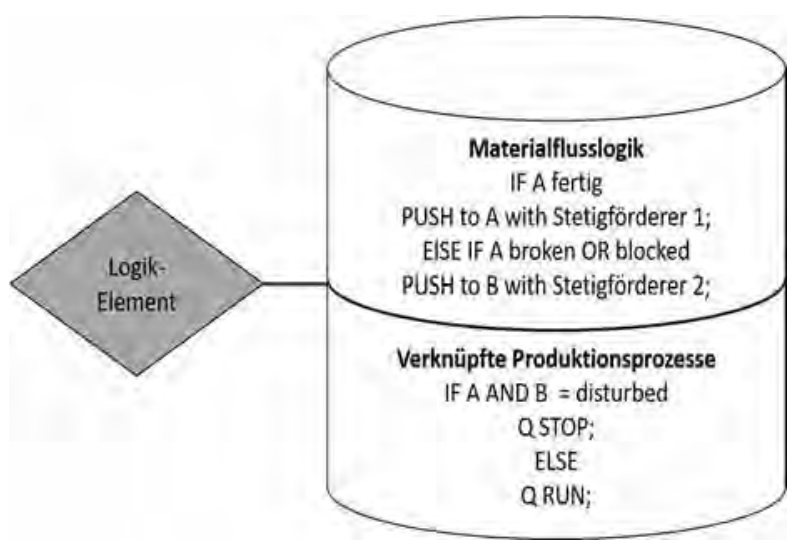

Abbildung 10: Beispiel zur Abbildung der Materiaflusslogik

Beispielsweise kann nach dem Montageprozess (Maschine Q) das Produkt auf Prozess A oder B geprüft werden. Der reguläre Materialfluss erfolgt über eine direkte Verbindung von Q nach A durch den Stetigförderer 
1 (Förderband). Wenn Prozess A ausfällt oder blockiert ist, wird das Produkt durch Stetigförderer 2 (Förderband) nach B transportiert. Sind Prozess A und B ausgefallen, stoppt Prozess Q.

Produkt. Die WSM analysiert den Wertstrom eines diskreten Produkts oder Produkttyps [2]. Für eine realistische Wertstromsimulation müssen verschiedene Produkte mit unterschiedlichen Zykluszeiten und Verhaltensweisen berücksichtigt werden. Dies ermöglicht eine Darstellung von Wertströmen und Montageprozessen mit unterschiedlichen Einheiten in Abhängigkeit von verschiedenen Produktionsaufträgen. Realisiert wird dies durch die Abbildung des Produktes mit spezifischen Attributen, z. B. der Prozessablaufliste einschließlich der Informationen über Quellen und Senken sowie des spezifischen Stückgutabstands. Das produktspezifische Attribut wird hierbei am jeweiligen Prozess abgefragt, was zu unterschiedlichen Ereignissen führt (Abbildung 11).

\begin{tabular}{l}
\hline Produktname \\
\hline Produktfamilie \\
\hline Losgrößc \\
\hline Prozessablauf: \\
- Prozcss Q \\
- Prozess A v B \\
\hline Stückgulabstand \\
\hline
\end{tabular}

Abbildung 11: Darstellung eines Produktes

\section{Referenzmodell basierend auf der WSM}

Die Vorteile des WSM, der DES und des RM sollen miteinander kombiniert werden. Dazu gehören die Transparenz des WSM, die Dynamik der DES und der reduzierte Modellierungsaufwand durch ein RM.

Darüber hinaus sollten für jeden Prozess Daten wie Zyklus und Transportzeit berücksichtigt werden. Ein Zeitdiagramm der Zyklus- und Transportzeiten ermöglicht die Erkennung von Engpässen. Abbildung 12 zeigt - basierend auf dem vorherigen Beispiel - wie ein Simulationsmodell, welches mit dem auf der Wertstromsimulation basierenden Referenzmodell modelliert wurde, aussehen würde.

Zusätzlich zur Analyse der WSM-Elemente (Materialflusslogik und Produkt) für das Referenzmodell ist eine Kosten-Nutzen-Bewertung erforderlich, um den Aufwand für die Modellierung mit dem Referenzmodell auf der Grundlage der Wertstromsimulation zu bestimmen. Diese Evaluierung und die Aspekte der Abbildung von Beständen und Puffern, die Integration des Zeitdiagramms und die Definition der notwendigen Ergebnisse (wie Durchsatz und Vorlaufzeit) werden in der weiteren Forschung betrachtet werden.

\section{Zusammenfassung und Ausblick}

Die Etablierung der Simulation in der Angebotsphase bietet eine Verbesserung für Unternehmen, die Produktionssysteme mit komplexen Materialflusssystemen planen. Mögliche Planungsfehler können früher erkannt werden, was die Wettbewerbsfähigkeit erhöht. Dies bildet die Grundlage für eine sichere und korrekte Planung sowie für eine exakte Angebotserstellung.

Eine wichtige Voraussetzung ist, dass der Modellierungsaufwand und die damit verbundene Vorlaufzeit der Angebotserstellung gering bleiben müssen. Die WSM ermöglicht hierzu eine transparente und übersichtliche Darstellung aller für die Herstellung eines Produktes erforderlichen Produktionsprozesse in Verbindung mit den entsprechenden Material- und Informationsflüssen [2]. Die Simulation ermöglicht eine exakte Abbildung dynamischer Systeme im Wertstrom [4]. Dies motivierte die Idee, VSM und Simulation zu kombinieren. Die Nachteile der Simulation sind der hohe Modellierungsaufwand sowie der intensive Ressourcenbedarf, der mit einem wertstromspezifischen Referenzmodell auf ein akzeptables Maß reduziert werden soll. Dieses Referenzmodell, das auf der Wertstromsimulation basiert, soll sich in den Hauptmerkmalen seiner Anwendung nicht von der herkömmlichen statischen WSM unterscheiden. Für den Aufbau des Referenzmodells werden die Grundelemente des WSM analysiert und um dynamische Aspekte erweitert.

Der Detaillierungsgrad des Referenzmodells - insbesondere zur Abbildung der Materialflusslogik - und seine Kosten-Nutzen-Bewertung werden in weiteren Untersuchungen erarbeitet.

\section{Danksagung}

Das Forschungsprojekt wurde von der Europäischen Union aus dem Europäischen Fonds für regionale Entwicklung sowie vom Land Rheinland-Pfalz gefördert, in Kooperation mit dem Industriepartner AtlanticC GmbH in Bernkastel-Kues. 


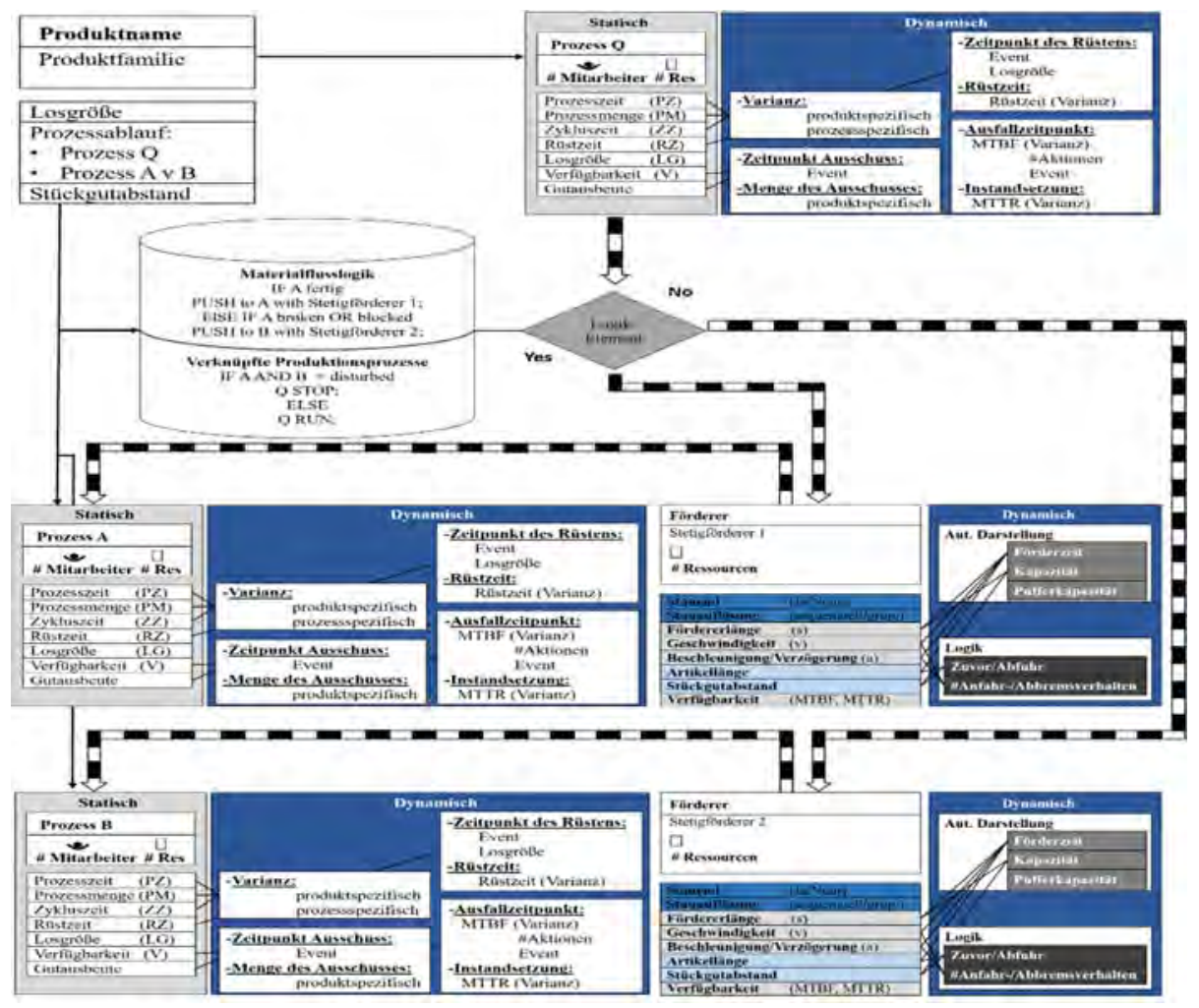

Abbildung 12: Simulation mit dem RM basierend auf Wertstromsimulation

\section{References}

[1] Friedland R, Kühling M. Referenzmodelle für Fertigungssysteme. In Wenzel S., ed. Referenzmodelle für die Simulation in Produktion und Logistik. Erlangen: SCS; 2000.

[2] Erlach K. Wertstromdesign: Der Weg zur schlanken Fabrik. 2nd ed. Berlin: Springer; 2010.

[3] Luger A, Winkler H. Von der Wertstromanalyse zum Wertstrommanagement. ZWF. 2017; 112 (4), 261-265.

[4] Gutenschwager K, Rabe M, Spieckermann S, Wenzel S. Simulation in Produktion und Logistik: Grundlagen und Anwendung. Berlin: Springer; 2017.

[5] VDI-Richtlinie 3633: Simulation von Logistik-, Materialfluss und Produktionssystemen - Blatt 1. Berlin: Beuth, 2014.

[6] Klinger A, Wenzel S. Referenzmodelle - Begriffsbestimmung und Klassifizierung. In Wenzel S., ed. Referenzmodelle für die Simulation in Produktion und Logistik. Erlangen: SCS; 2000.

[7] Schütte R. Grundsätze ordnungsmäßiger Referenzmodellierung: Konstruktion konfigurations- und anpassungsorientierter Modelle. Wiesbaden: Springer; 1998.

[8] Drees J. Neue Perspektiven für die WSM: Wertstrom Management 4.0. ZWF. 2018; 113 (9), 605-609.

[9] Rabe M, Wincheringer W, Sohny T. Reference model based on value stream simulation for the evaluation of production systems in the bidding phase. RIRL-SCM; 2020, to be published.
[10] VDI-Richtlinie 2689: Leitfaden für Materialflussuntersuchungen. 2019.

[11] Knössl T. Logistikorientierte Wertstromanalyse. In Günther W, Boppert J., editors. Lean Logistics: Methodisches Vorgehen und praktische Anwendung in der Automobilindustrie. Berlin: Springer; 2013.

[12] Ten Hompel M, Schmidt T, Dregge J. Materialflusssysteme: Förder- und Lagertechnik. Berlin: Springer; 2018.

[13] VDI-Richtiline 3978: Durchsatz und Spielzeitberechnungen in Stückgut-Fördersystemen. 2018.

[14] Gudehus, T. Logistik 2: Netzwerke, Systeme und Lieferketten. Berlin, Heidelberg: Springer; 2012.

[15] Lippolt CR. Spielzeiten in Hochregallagern mit doppeltiefer Lagerung. Dissertation TH Karlsruhe. 2003.

[16] Jung E, ten Hompel M. Analytische Stauprognose in Stetigfördersystemen im Rahmen der Systemplanung. Logistics Journal: Proceedings - ISSN 2192-9084; 2013.

[17] Lienert T, Fottner J. Entwicklung einer generischen Simulationsmethode für das zeitfensterbasierte Routing Fahrerloser Transportfahrzeuge. Logistics Journal: Proceedings - ISSN 2192-9084; 2013.

[18] Ullrich G. Fahrerlose Transportsysteme Eine Fibel - mit Praxisanwendungen - zur Technik - für die Planung. 2nd ed. Wiesbaden: Springer; 2014.

[19] Türck S, Weimer A, Schubert L, Drees J. Dynamische Simulation von Wertströmen. ZWF. 2014; 109 (11), 839842. 